\title{
QUANTIFYING THE HEATING SOURCES FOR MID-INFRARED DUST EMISSIONS IN GALAXIES: THE CASE OF M 81*
}

\author{
N. Lu ${ }^{1}$, G. J. Bendo ${ }^{2}$, A. Boselli ${ }^{3}$, M. BAes ${ }^{4}$, H. Wu ${ }^{5}$, S. C. MAdDen ${ }^{6}$, I. De LoOze ${ }^{4}$, A. Rémy-Ruyer ${ }^{6,7}$,

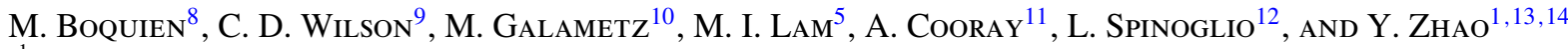 \\ ${ }^{1}$ Infrared Processing and Analysis Center, California Institute of Technology, MS 100-22, Pasadena, CA 91125, USA; lu@ipac.caltech.edu \\ 2 Jordrell Bank Center for Astrophysics, School of Physics and Astronomy, University of Manchester, Oxford Road, Manchester M13 9PL, UK \\ ${ }^{3}$ Laboratoire d'Astrophysique de Marseille-LAM, Université d'Aix-Marseille and CNRS, UMR7326, 38 rue F. Joliot-Curie, F-13388 Marseille Cedex 13, France \\ ${ }^{4}$ Sterrenkundig Observatorium, Universiteit Gent, Krijgslaan 281 S9, B-9000 Gent, Belgium \\ ${ }^{5}$ Key Laboratory of Optical Astronomy, National Astronomical Observatories, Chinese Academy of Sciences, A20 Datun Road, Beijing 100012, China \\ ${ }^{6}$ Laboratoire AIM, CEA, Université Paris VII, IRFU/Service d'Ástrophysique, Bat. 709, F-91191 Gif-sur-Yvette, France \\ ${ }^{7}$ Institut d'Astrophysique Spatiale, CNRS, UMR8617, F-91405 Orsay, France \\ ${ }^{8}$ Institute of Astronomy, University of Cambridge, Madingley Road, Cambridge, CB3 0HA, UK \\ ${ }^{9}$ Department of Physics and Astronomy, McMaster University, Hamilton, Ontario L8S 4M1, Canada \\ ${ }^{10}$ European Southern Observatory, Karl-Schwarzschild-Str. 2, D-85748 Garching-bei-Mnchen, Germany \\ ${ }^{11}$ Department of Physics and Astronomy, University of California, Irvine, CA 92697, USA \\ 12 Istituto di Astrofisica e Planetologia Spaziali, INAF, Via Fosso del Cavaliere 100, I-00133 Roma, Italy \\ ${ }^{13}$ Purple Mountain Observatory, Chinese Academy of Sciences, Nanjing 210008, China \\ ${ }^{14}$ Key Laboratory of Radio Astronomy, Chinese Academy of Sciences, Nanjing 210008, China \\ Received 2014 June 3; accepted 2014 October 13; published 2014 December 8
}

\begin{abstract}
With the newly available photometric images at 250 and $500 \mu \mathrm{m}$ from the Herschel Space Observatory, we study quantitative correlations over a sub-kiloparsec scale among three distinct emission components in the interstellar medium of the nearby spiral galaxy M 81 (NGC 3031): (1) $I_{8}$ or $I_{24}$, the surface brightness of the mid-infrared emission observed in the Spitzer Space Telescope 8 or $24 \mu \mathrm{m}$ band, with $I_{8}$ and $I_{24}$ being dominated by the emissions from polycyclic aromatic hydrocarbons (PAHs) and very small grains (VSGs) of dust, respectively; (2) $I_{500}$, that of the cold dust continuum emission in the Herschel Space Observatory $500 \mu \mathrm{m}$ band, dominated by the emission from large dust grains heated by evolved stars; and (3) $I_{\mathrm{H} \alpha}$, a nominal surface brightness of the H $\alpha$ line emission, from gas ionized by newly formed massive stars. The results from our correlation study, free from any assumption on or modeling of dust emissivity law or dust temperatures, present solid evidence for significant heating of PAHs and VSGs by evolved stars. In the case of M 81, about 67\% (48\%) of the $8 \mu \mathrm{m}(24 \mu \mathrm{m})$ emission derives its heating from evolved stars, with the remainder attributed to radiation heating associated with ionizing stars.
\end{abstract}

Key words: galaxies: individual (M 81) - galaxies: ISM - galaxies: star formation - infrared: galaxies infrared: ISM - ISM: lines and bands

\section{INTRODUCTION}

Mid-infrared ( 5 to $\sim 40 \mu \mathrm{m}$ ) emission in the interstellar medium (ISM) of normal disk galaxies is dominated by (1) broad emission features over $5-15 \mu \mathrm{m}$, with the main features at 6.2, 7.7, 8.6, 11.3, $12.7 \mu \mathrm{m}$ (e.g., Lu et al. 2003), and (2) a hot dust continuum longward of $\sim 15 \mu \mathrm{m}$, which was first detected by the Infrared Astronomical Satellite (IRAS) in our own Galaxy (e.g., Castelaz et al. 1987). While (1) is widely believed to be the emission bands from polycyclic aromatic hydrocarbon molecules (PAHs) of sizes up to $\sim 20 \AA$ (Puget \& Léger 1989; Allamandola et al. 1989), (2) is commonly attributed to the emission from very small grains (VSGs) of dust of sizes of $\sim 20$ to $\sim 100 \AA$ (Puget \& Léger 1989; Désert et al. 1990). PAHs and VSGs are both heated transiently through the absorption of single UV/optical photons. For a given PAH or VSG population and a largely fixed shape of the heating radiation field, the spectral shape of the PAH or VSG emission should be largely independent of the intensity of the radiation field (e.g., Draine \& Li 2007). As a result, the total flux integrated over their spectrum scales linearly with a flux density sampled at any frequency over the spectrum. A practical choice is to use

\footnotetext{
* Herschel is an ESA space observatory with science instruments provided by European-led Principal Investigator consortia and with important participation from NASA.
}

the flux densities measured with the Spitzer Space Telescope (Spitzer) Infrared Array Camera (IRAC) in its $8 \mu \mathrm{m}$ band and Multiband Imaging Photometer for Spitzer (MIPS) at $24 \mu \mathrm{m}$ to represent the spectrally integrated fluxes of the PAH and VSG emissions, respectively. We note that the picture described above may not be fully applicable to all types of galaxies. For example, for early-type galaxies, the stellar emission may be significant at $8 \mu \mathrm{m}$; for starburst galaxies, the emission from large dust grains may start to contribute at $24 \mu \mathrm{m}$ when their thermal equilibrium temperature reaches above $\sim 45 \mathrm{~K}$ (e.g., Boulanger et al. 1988).

Both the 8 and $24 \mu \mathrm{m}$ flux densities have been widely used as tracers of the current star formation rate (SFR), both in the local universe and at high redshifts. Of these two, the $8 \mu \mathrm{m}$ flux is more controversial as to whether the heating of the underlying PAHs is primarily derived from current star formation: on one hand, the PAH emission has been shown to correlate with a known SFR tracer such as the $\mathrm{H} \alpha$ line emission, mostly based on flux-to-flux correlations (e.g., Roussel et al. 2001; Förster Schreiber et al. 2004; Wu et al. 2005; Zhu et al. 2008); on the other hand, it has been well known from the Cosmic Background Explorer (e.g., Dwek et al. 1997), the Infrared Space Observatory (ISO; e.g., Lemke et al. 1998; Uchida et al. 1998), the Infrared Telescope in Space (e.g., Chan et al. 2001), and Spitzer (e.g., Lu 2004) that, in our own Galaxy, the PAH emission arises plentifully in the general ISM and discrete sources devoid of a strong UV radiation field. Additional 
support for a possible PAH component unrelated to current star formation in normal spirals includes striking similarity in the large-scale intensity maps of the PAH emission and the cold dust continuum emission at $850 \mu \mathrm{m}$ (Haas et al. 2002), results from various surface brightness correlation studies based on ISO (e.g., Boselli et al. 2004; Irwin \& Madden 2006) and Spitzer (e.g., Bendo et al. 2008), as well as the fact that the $8 \mu \mathrm{m}$ or the $24 \mu \mathrm{m}$ to the $\mathrm{H} \alpha$ flux density ratio differs significantly between within or near individual $\mathrm{H}$ II regions and over galaxies as a whole (e.g., Calzetti et al. 2007; Zhu et al. 2008; Kennicutt et al. 2009).

A framework that is probably acceptable to both schools of opposite views is such that a line-of-sight flux of the PAH emission normally consists of two components: a "warm" component powered by current star formation and a "cold" diffuse component powered by the general interstellar radiation field dominated by evolved stars. The controversy described above stems largely from the fact that it is practically impossible to unambiguously separate these two components in a two-dimensional galaxy image as they are not spatially anti-correlated because the Kennicutt-Schmidt law (Schmidt 1959; Kennicutt 1998) dictates that the dust/gas distribution should concentrate around star forming regions even if much of the surrounding emission from a particular dust population may not be directly related to the current star formation. One can reduce (but not totally eliminate) this "degeneracy" by studying dust emission maps at finer linear scales.

Recent images from the Herschel Space Observatory (Herschel; Pilbratt et al. 2010) provided cold dust emission maps at linear resolutions as low as $\sim 100 \mathrm{pc}$ in some nearby disk galaxies, such as M 33 (Boquien et al. 2011; Xilouris et al. 2012; Calapa et al. 2014) and NGC 2403 (Jones et al. 2014). By correlating the $8 \mu \mathrm{m}$ emission with a submillimeter flux density of the cold dust emission or the stellar continuum flux density at $3.6 \mu \mathrm{m}$, these studies generally favor the picture that most of the PAH emission in a normal disk galaxy is not directly related to the ongoing star formation (but also see Crocker et al. 2013). Most of the aforementioned studies inherit one or both of the following limitations: they either used a flux density at a fixed wavelength (e.g., $250 \mu \mathrm{m}$ ) to characterize the cold dust emission or a near-IR stellar continuum (e.g., at $3.6 \mu \mathrm{m}$ ) to represent the heating radiation field of non-ionizing stars. The former could introduce biases as the cold dust emission is always in a thermal equilibrium with the heating radiation field and a monochromatic flux density at a fixed wavelength represents a variable fraction of the total (i.e., frequency-integrated) cold dust emission across a galaxy disk; the latter may bias against the type of nonionizing stars that are most efficient in dust heating when it is used across the entire galaxy.

In this paper, we use an alternative, "two-component" analysis to separate the warm and cold dust emission components across a galaxy disk. This method is in principle valid for galaxy images with any angular resolution and free from either of the potential biases mentioned above. Our goal is to answer whether or under what condition the warm PAH component becomes dominant for a star forming galaxy as a whole. Lu \& Helou (2008) pioneered this approach using a sample of IRAS galaxies with available $850 \mu \mathrm{m}$ fluxes. Letting $F_{\text {warm }}$ and $F_{\text {cold }}$ be the spectrally integrated fluxes of the corresponding warm and cold emissions from large dust grains in the far-infrared (FIR), one can write

$$
F_{\mathrm{PAH}}=A F_{\mathrm{warm}}+B F_{\text {cold }},
$$

where $A$ and $B$ are two scaling factors to be determined observationally. $F_{\mathrm{PAH}}$ is the spectrally integrated flux of the PAH emission, which is simply proportional to the flux of any combination of the major PAH bands. Strictly speaking, both $A$ and $B$, which measure the PAH emission relative to the large dust grain emission, are constant under the conditions of (1) a fixed dust mass spectrum (i.e., a fixed PAH-to-large dust grain abundance ratio) and (2) a more or less fixed shape of the dust heating radiation field in each of the cold and warm components (e.g., of similar hardness). In reality, both (1) and (2) can certainly vary from one galaxy to another and may even vary modestly across a galaxy disk. Nevertheless, it is still meaningful to derive values of $A$ and $B$ averaged over a galaxy disk or galaxies of some similar properties (e.g., comparable FIR dust color temperatures).

Replacing $F_{\text {PAH }}$ with the IRAC $8 \mu \mathrm{m}$ flux density and deriving $F_{\text {warm }}$ and $F_{\text {cold }}$ crudely from the IRAS 60 and $100 \mu \mathrm{m}$ and the $850 \mu$ m fluxes, Lu \& Helou (2008) found statistically that the cold PAH component is usually the dominant one except for very actively star forming galaxies (i.e., those with an IRAS 60-to- $100 \mu \mathrm{m}$ flux density ratio (hereafter referred to as FIR color $\left.), f_{v}(60 \mu \mathrm{m}) / f_{v}(100 \mu \mathrm{m}) \gtrsim 0.6\right]$. One implication from this study is that the fractional $\mathrm{PAH}$ emission from the star formation component varies from galaxy to galaxy. If further confirmed, this may pose an apparent conflict with the constant star formation fraction of the PAH emission used in the composite $\mathrm{H} \alpha$ and $8 \mu \mathrm{m} \mathrm{SFR}$ tracer for galaxies (e.g., Kennicutt et al. 2009).

With the unprecedented sensitivity and improved spatial resolution of the Spectral and Photometric Imaging REceiver (SPIRE; Griffin et al. 2010) on board Herschel, images of nearby spiral galaxies at 250, 350, and $500 \mu \mathrm{m}$ with sub-kiloparsec linear resolutions have become available. For normal spiral galaxies, these images are usually dominated by the cold dust emission powered by evolved stars (e.g., Bendo et al. 2012). This makes it possible to carry out a two-component analysis over the disks of individual galaxies at a linear resolution limited by the SPIRE images, not only for the PAH emission, but also for the VSG dust emission. In this paper, we present a case study on M 81 (NGC 3031), one of the nearest normal spiral galaxies. M 81 has a morphological type SA(s)ab and an optical disk of $26^{\prime} .9 \times 14^{\prime} .1$ (i.e., $R_{25}$ in B; de Vaucouleurs et al. 1991), inclined at $59^{\circ}$ from face-on (de Blok et al. 2008). At its distance of 3.63 Mpc (Karachentsev et al. 2002), the 36.'3 beam of the FWHM of the SPIRE $500 \mu \mathrm{m}$ band corresponds to $0.64 \mathrm{kpc}$. The galaxy is relatively infrared quiescent with an FIR color of $\sim 0.26$ and has an IRAS FIR luminosity of $3 \times 10^{9} L_{\odot}$ (Rice et al. 1988). M 81 is known to host a weak nuclear LINER (Heckman 1980), which may have some effect on the nuclear infrared emission. However, this should be limited to within one SPIRE $500 \mu \mathrm{m}$ beam size (Bendo et al. 2012) and have little effect on our statistical results that are dominated by the galaxy disk. In the remainder of this paper, we lay out our method in Section 2, describe the data used in Section 3, present our correlation analysis results in Section 4, and assess potential systematics in Section 5. We then discuss some astronomical implications in Section 6 and finally summarize our main conclusions in Section 7.

\section{METHOD}

We can rewrite Equation (1) in terms of surface brightnesses. For the PAH emission, we have

$$
I_{8}=a I_{\mathrm{H} \alpha}+b K(T) I_{500},
$$


where $I_{8}$ is the surface brightness of the PAH emission in the Spitzer IRAC $8 \mu \mathrm{m}$ band, $I_{\mathrm{H} \alpha}$ is the nominal surface brightness of the $\mathrm{H} \alpha$ line emission, $I_{500}$ is the surface brightness of the dust continuum emission in the SPIRE $500 \mu \mathrm{m}$ band, and the factor $K(T)$ is defined as such that

$$
K(T) I_{500}=\int_{0}^{+\infty} I_{\nu} d v
$$

where the integration is performed in frequency over the cold dust emission spectrum of temperature $T$. While Equation (3) illustrates the definition of $K(T)$ in the context of a singletemperature dust emission, $K(T)$ itself is a generic K-correction factor that is valid even if the cold dust emission involves multiple dust temperatures and/or emissivities.

The first term on the right-hand side of Equation (2) represents the warm component of the PAH emission, under the implicit assumption that the $\mathrm{H} \alpha$ line emission scales well locally with the far-UV light that dominates the heating of the warm dust. This should be a fairly good assumption for disk galaxies of normal gas surface brightness and metallicity, such as M81, as the dust and gas are well mixed and the dust absorption of far-UV light is very efficient. Observationally, this is backed up by the nearly one-to-one correlation observed between the $\mathrm{PAH}$ and $\mathrm{H} \alpha$ surface brightnesses for individual $\mathrm{H}$ II regions in normal galaxies (e.g., Calzetti et al. 2007). (We revisit this and other potential systematics in our method in Section 5.)

The second term on the right-hand side of Equation (2) represents the cold $\mathrm{PAH}$ component, under the assumption that $I_{500}$ is dominated by the continuum emission of large dust grains heated mainly by evolved stars. We show in Section 4 that this assumption should be a good one in the case of M81, a conclusion that was also reached independently by Bendo et al. (2012). The factor $K(T)$ in Equation (2) depends on both cold dust temperature $T$ and dust emissivity $\beta$. Using the data from and the spectral-energy-distribution-(SED)-fitting methods applied by Bendo et al. (2010), we see that, at radii greater than $3 \mathrm{kpc}$ in M 81, the cold dust heated by the evolved stars has temperatures of $15-20 \mathrm{~K}$ and $\beta$ values of approximately 2 . For these SED fits, $I_{250} / I_{500}$ increases from $\sim 4$ to $\sim 6$ with $K(20 \mathrm{~K}) / K(15 \mathrm{~K}) \approx 3$, where $I_{250}$ is the surface brightness in the SPIRE $250 \mu \mathrm{m}$ band. It will become clear in Section 4 that this empirical approach (as opposed to a full modeling of the quantity $\int I_{\nu} d \nu$ ) has the advantage that our results are largely free from any assumption on $T$ or $\beta$. The two constant scaling factors in Equation (2), $a$ and $b$, are to be determined from the data.

Similarly we can also write $I_{24}$, the surface brightness of the dust emission in the Spitzer $24 \mu \mathrm{m}$ band, as follows:

$$
I_{24}=c I_{\mathrm{H} \alpha}+d K(T) I_{500},
$$

where $c$ and $d$ are two appropriate scaling factors to be determined.

\section{DATA}

\subsection{Herschel Images at 250 and $500 \mu \mathrm{m}$}

The SPIRE images used in this paper are the same as those given in Bendo et al. (2012) and were from a SPIRE observation $($ ObsID $=1342185538)$ under the Very Nearby Galaxy Survey project (PI: C. D. Wilson). The observation, data reduction, and image construction were described in detail in Bendo et al. (2012). The original images have a size of $40^{\prime} \times 40^{\prime}$ and a
Table 1

Image Characteristics

\begin{tabular}{lcccccc}
\hline \hline $\begin{array}{l}\text { Waveband } \\
(\mu \mathrm{m})\end{array}$ & 3.6 & 8 & 24 & 250 & 500 & $\mathrm{H} \alpha$ \\
\hline FWHM (arcsec) & $1.7^{\mathrm{a}}$ & $1.9^{\mathrm{a}}$ & $6.0^{\mathrm{a}}$ & $18.2^{\mathrm{b}}$ & $36.3^{\mathrm{b}}$ & 1.0 \\
Pixel size (arcsec) & 0.75 & 0.75 & 2.45 & 6 & 14 & 2.03 \\
\hline
\end{tabular}

Notes.

${ }^{a}$ See the Spitzer IRAC or MIPS Instrument Handbook.

${ }^{\mathrm{b}}$ See the Herschel SPIRE Handbook.

flux calibration uncertainty of $\lesssim 10 \%$ (i.e., Herschel Interactive Processing Environment or HIPE, version 5). The overall SPIRE flux calibration accuracy has since improved. Nevertheless, this has no effect on the correlation analysis performed in this paper as the SPIRE detector nonlinearity correction remained unchanged. The adopted spatial resolutions of these images are given in Table 1.

The surrounding regions of M 81 show patchy cirrus dust emission from our Galaxy (Davies et al. 2010). These extended emissions pose some limits on the accuracy of sky subtraction. Nevertheless, a mean residual sky background was carefully removed from each SPIRE image. We further smoothed the $250 \mu \mathrm{m}$ image to the same spatial resolution of the $500 \mu \mathrm{m}$ image using a Gaussian kernel with a FWHM of 31".4 [= $\left.\left(36^{\prime \prime} 3^{2}-18^{\prime \prime} \cdot 2^{2}\right)^{1 / 2}\right]$. The smoothed $250 \mu \mathrm{m}$ image was then resampled to the same $14^{\prime \prime}$ pixel scale as the $500 \mu \mathrm{m}$ image. No color correction was applied to either image.

\subsection{Spitzer Images at 8 and $24 \mu \mathrm{m}$}

The IRAC images at 3.6 and $8.0 \mu \mathrm{m}$ and MIPS image at $24 \mu \mathrm{m}$ used in this paper are the released mosaic images from the Spitzer Infrared Nearby Galaxies Survey project (SINGS $^{15}$; Kennicutt et al. 2003). The images we used here are similar to those in Bendo et al. (2008). We further multiplied the 3.6 and $8 \mu \mathrm{m}$ images by 0.91 and 0.74 (see the IRAC Instrument manual, Table 4.8), respectively, to be consistent with the extendedsource flux calibration.

The resulting $3.6 \mu \mathrm{m}$ image was smoothed to the spatial resolution of the $8 \mu \mathrm{m}$ image using a Gaussian kernel with a FWHM of $0.85^{\prime \prime}\left[=\left(1^{\prime \prime} .9^{2}-1^{\prime \prime} .7^{2}\right)^{1 / 2}\right]$ (see Table 1$)$. The smoothed $3.6 \mu \mathrm{m}$ image was used to remove the stellar contribution in the $8 \mu \mathrm{m}$ image as follows:

$$
I_{8}=\left(I_{8}^{\text {tot }}-0.232 I_{3.6}\right) / 0.99,
$$

where $I_{8}^{\text {tot }}$ and $I_{3.6}$ represent the observed surface brightnesses at 8 and $3.6 \mu \mathrm{m}$, respectively. The term $0.232 I_{3.6}$ (see Helou et al. 2004) in Equation (5) accounts for the stellar flux at $8 \mu \mathrm{m}$ if all of $I_{3.6}$ is of stellar origin. In reality, the $3.6 \mu \mathrm{m}$ flux in disk galaxies is contaminated by a dust continuum (e.g., Lu et al. 2003; Mentuch et al. 2010). This contamination is equal to $\sim 5 \% I_{8}$ (Lu 2004). The denominator on the right-hand side of Equation (5) corrects for this. The non-stellar $8 \mu \mathrm{m}$ image was subsequently smoothed to the same spatial resolution as the SPIRE $500 \mu \mathrm{m}$ image, and resampled to a $14^{\prime \prime}$ pixel scale. This smoothing process used a Gaussian kernel with a FWHM of $36^{\prime \prime} .2\left[=\left(36^{\prime \prime} \cdot 3^{2}-1^{\prime \prime} \cdot 9^{2}\right)^{1 / 2}\right]$.

As an early-type disk galaxy, M 81 has a large bulge, which has some stellar contamination even at $24 \mu \mathrm{m}$, especially in

\footnotetext{
15 The description of these images can be found at http://irsa.ipac.caltech.edu/data/SPITZER/SINGS/.
} 
the low surface brightness regions between spiral arms. This stellar contamination was removed in the following way. First, the $3.6 \mu \mathrm{m}$ image was smoothed to match the point-spread function (PSF) of the $24 \mu \mathrm{m}$ image using an IRAC 3.6 to MIPS $24 \mu \mathrm{m}$ convolution kernel $^{16}$ appropriate for a $100 \mathrm{~K}$ blackbody spectrum (see Gordon et al. 2008). This kernel function takes into consideration the fact that the MIPS $24 \mu \mathrm{m}$ PSF deviates from a Gaussian profile. The kernel was first azimuthally averaged before it was convolved with the IRAC $3.6 \mu \mathrm{m}$ image. The convolved image was then multiplied by a factor $0.0258\left[=0.232(8 \mu \mathrm{m} / 24 \mu \mathrm{m})^{2}\right.$, with the factor 0.232 from Equation (5)] and subsequently subtracted from the $24 \mu \mathrm{m}$ image. The amount of the subtraction accounts for $1 \%-2 \%$ of $I_{24}$ along the spiral arms, but could be as large as $10 \%-15 \%$ of $I_{24}$ in the low surface brightness regions between the outer spiral arms. The resulting dust-only $24 \mu \mathrm{m}$ image was further smoothed to the resolution of the SPIRE $500 \mu \mathrm{m}$ band using the same 24-to-500 $\mu \mathrm{m}$ convolution kernel as in Bendo et al. (2012). Finally, the resulting $24 \mu \mathrm{m}$ image was also resampled to a $14^{\prime \prime}$ pixel scale.

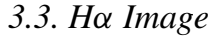

The $\mathrm{H} \alpha$ image was taken, via the NASA/IPAC Extragalactic Database (NED), from Hoopes et al. (2001) with the original image presented in Greenawalt et al. (1998). The image was taken in a filter of $75 \AA$ in bandwidth, which encompasses the [N II] doublet. In M 81, the metallicity varies (mainly radially) slowly across the outer disk (i.e., over a face-on radius between $\sim 5$ and $\sim 11 \mathrm{kpc}$ ), but increases more rapidly toward the nucleus within the bulge (Garnett \& Shields 1987). We assumed a constant ratio of $[\mathrm{N} I \mathrm{II}] / \mathrm{H} \alpha$ for the disk and did not subtract [N II] from the $\mathrm{H} \alpha$ image. We discuss in Section 5 the potential systematic effect on our results from possibly higher [N II]/ $\mathrm{H} \alpha$ ratios within the bulge. The image was rotated to the correct orientation, smoothed to the resolution of the SPIRE $500 \mu \mathrm{m}$ image using a simple Gaussian kernel based on the FWHM values given in Table 1, and resampled to our final pixel size of $14^{\prime \prime}$. There are other $\mathrm{H} \alpha$ images available (e.g., Boselli \& Gavazzi 2002; Sánchez-Gallego et al. 2012). They are quantitatively very similar to the one we used in the paper, at least outside the nucleus.

The original $\mathrm{H} \alpha$ image has a flux unit of $2.0 \times$ $10^{-21} \mathrm{~W} / \mathrm{m}^{2} / \operatorname{arcsec}^{2}$ (Greenawalt et al. 1998). We converted the image into a nominal surface brightness by dividing each image pixel value by the $\mathrm{H} \alpha$ rest-frame frequency of $4.57 \times 10^{14} \mathrm{~Hz}$. No correction for either foreground or internal extinction was done in this work. The foreground and average internal disk extinctions for M 81 are on the order of $A_{V} \sim 0.2$ and $0.4 \mathrm{mag}$, respectively (e.g., Perelmuter \& Racine 1995; Schroder et al. 2002) and should have no effect on our analysis results. On the other hand, a significant patchiness in the internal extinction could have some systematic effect on our analysis. The inferred internal extinctions toward a large number of globular clusters across the entire disk of M81 show only a moderate variation, within a factor of two of the mean value (Schroder et al. 2002). Nevertheless, local internal extinction could be significantly higher toward prominent star forming regions (e.g., Kaufman et al. 1989), resulting in a systematic difference in the attenuation of the $\mathrm{H} \alpha$ line flux between star forming and diffuse regions. We come back to discuss its effect on our results in Section 5 .

\footnotetext{
16 Available at http://dirty.as.arizona.edu/ kgordon/mips/conv_psfs/
} conv_psfs.html.
It has been known that star forming disk galaxies show substantial "diffuse" ionized gas and $\mathrm{H} \alpha$ line emission outside bright H II regions (e.g., Reynolds 1991; Walterbos \& Braun 1994; Haffner et al. 2009 and references therein). It is generally believed that this diffuse $\mathrm{H} \alpha$ emission is associated with ionizing radiation that leaks out of HiI regions (e.g., Mathis 1986; Ferguson et al. 1996; Wood \& Reynolds 1999), although some dust scattering of $\mathrm{H} \alpha$ photons may also play a role at least at high galactic latitudes (e.g., Mattila et al. 2007; Witt et al. 2010). In this paper, we follow the notion that the diffuse $\mathrm{H} \alpha$ line flux originates from current star formation and that its associated local UV light powers a warm dust emission component.

All the final images were spatially co-aligned, and have a pixel scale of $14^{\prime \prime}$ and an image size of 76 pixels (east-west) times 117 pixels (north-south). The surface brightness units are $\mathrm{Jy} \mathrm{pixel}^{-1}$ for all but the $\mathrm{H} \alpha$ image, for which the units are mJy pixel $^{-1}$. These images, except for the $250 \mu \mathrm{m}$ image, are shown in Figure 1, where only image pixels with a surface brightness three times above the corresponding sky noise are plotted. Both Herschel 250 and $500 \mu \mathrm{m}$ images can also be seen in Bendo et al. (2012).

\section{ANALYSIS}

\subsection{Surface Brightness Ratios}

On the surface, all four images in Figure 1 have similar appearances and trace each other well qualitatively, except for the nucleus, where it is relatively faint in the $500 \mu \mathrm{m}$ image. The ellipse shown in each image corresponds to a face-on angular radius, $R_{\text {face-on }}=644^{\prime \prime}\left(\sim 0.8 R_{25}\right)$, outside of which $I_{500}$ becomes mostly below $3 \sigma$. The apparent correlation across all the bands over much of the galaxy disk is, to a large extent, a result of the fact that dust, gas, and star distributions are all regulated by the spiral pattern. As a result, one can always find a flux-to-flux correlation to a certain degree between any two wavebands.

In Figure 2 we show surface brightness ratio images of $I_{8} / I_{\mathrm{H} \alpha}$, $I_{24} / I_{\mathrm{H} \alpha}, I_{\mathrm{H} \alpha} / I_{500}$, and $I_{250} / I_{500}$. The two ellipses shown in each image correspond to the face-on circles of $R_{\text {face-on }}=$ $250^{\prime \prime}$ and $644^{\prime \prime}$ (i.e., 4.4 and $11.3 \mathrm{kpc}$ at the galaxy distance), respectively. The inner radius was chosen for the following considerations: (1) there are no discreet $\mathrm{H}$ II regions within this radius (except for the nuclear region), and (2) in the outer disk, over $250^{\prime \prime}<R_{\text {face-on }}<644^{\prime \prime}$, the bright H II and diffuse regions occupy similar ranges in $K(T)$.

While the first three ratio images in Figure 2 are quite patchy, the image of $I_{250} / I_{500}$ is much more smooth, consistent with the claim by Bendo et al. $(2010,2012)$ that the dust emission over these wavelengths mostly derives its heating from evolved stars, which are also more smoothly distributed than ionizing stars across the galaxy disk. This is further supported by the radial color plots in Figure 3, where the azimuthal averages and sample standard deviations in individual radial bins are shown for the same four surface brightness ratios in Figure 2. The averages are normalized by the value in the inner most radial bin; the sample standard deviations are normalized by the actual (i.e., unnormalized) average radio in their corresponding radial bin. It is clear that the $I_{250} / I_{500}$ ratio is much smoother than the other three ratios in terms of both radial and azimuthal variations. Furthermore, the $I_{250} / I_{500}$ ratio shows only marginal change at most across any of those prominent disk $\mathrm{H}$ II regions visible between the two marked ellipses in the image of $I_{\mathrm{H} \alpha} / I_{500}$. We found that the reduction in $I_{250} / I_{500}$ from within one of these 

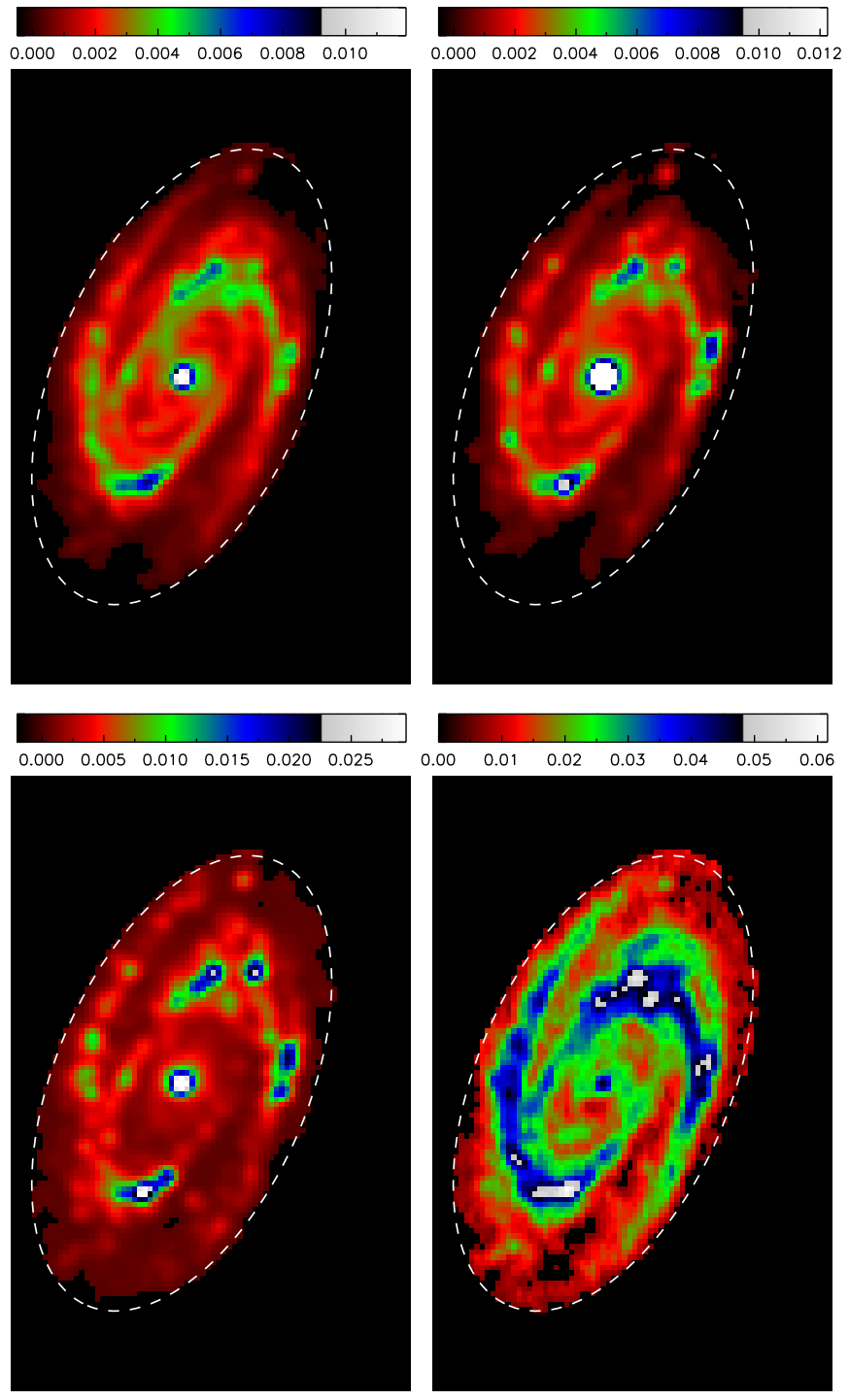

Figure 1. Surface brightness images in linear scale at $8 \mu \mathrm{m}$ (top left) and $24 \mu \mathrm{m}$ (top right) of the $\mathrm{H} \alpha$ line emission (bottom left) and at $500 \mu \mathrm{m}$ (bottom right). Each image is of 76 pixels (east-west) times 117 pixels (north-south), with north up and east to the left. These images, which have been co-aligned spatially, all have a spatial resolution of $\sim 36^{\prime \prime}$ and a pixel size of $14^{\prime \prime}$. The surface brightness is in $\mathrm{Jy} \mathrm{pixel}^{-1}$ (except for the $\mathrm{H} \alpha$ image, for which it is $\mathrm{mJy} \mathrm{pixel}^{-1}$ ) and color coded according to the color bar shown on top of the image. (Note that in order to show the disk structure, the inner galaxy nucleus is saturated in color in the $8 \mu \mathrm{m}, 24 \mu \mathrm{m}$, and $\mathrm{H} \alpha$ images.) Those image pixels with a surface brightness $\leqslant 3 \sigma$ are deemed unreliable and shown with a zero intensity here, where $\sigma$ is the sky noise in the image. The ellipse plotted in each image corresponds to a face-on circular aperture of $644^{\prime \prime}$ in radius.

$\mathrm{H}$ II regions to the general ISM just outside the $\mathrm{H}$ II region (but at the same galactocentric distance) is no more than $12 \%$.

Figure 4 plots the expected color change in $I_{250} / I_{500}$ as a function of the $250 \mu \mathrm{m}$ flux density ratio of a warm dust emission of $T_{\text {warm }}=30,40$, or $50 \mathrm{~K}$ to a cold dust component of $T_{\text {cold }}=16 \mathrm{~K}$. Such a cold dust component can reproduce well the observed median ratio of $I_{250} / I_{500}=4.5$ over the outer disk when the dust emissivity scales as $v^{2}$. Two-component SED fits to the FIR dust emission in normal galaxies generally result in $T_{\text {warm }}$ between 30 and $40 \mathrm{~K}$ (e.g., Bendo et al. 2003), which correspond to the region between the solid and dashed curves in Figure 4. These curves show that a $Y$-axis value of $1.14(=1 /(1 \%-12 \%)$; i.e., a $14 \% \mathrm{H}$ II region-related enhancement relative to the diffuse region just outside the
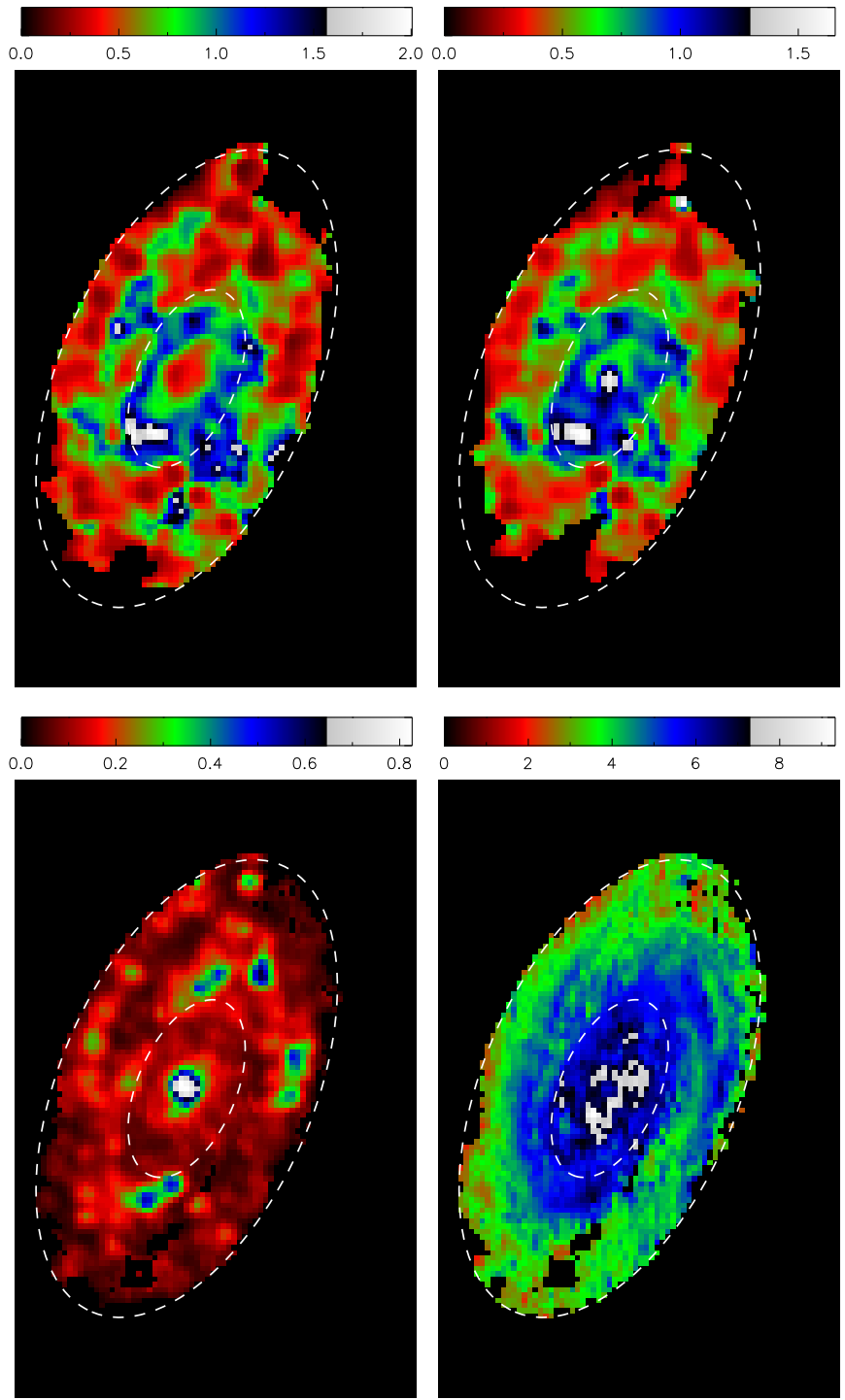

Figure 2. Similar to Figure 1, but for images of various surface brightness ratios: $I_{8} /\left(10^{3} I_{\mathrm{H} \alpha}\right)$ (top left), $I_{24} /\left(10^{3} I_{\mathrm{H} \alpha}\right)$ (top right), $\left(10^{3} I_{\mathrm{H} \alpha}\right) / I_{500}$ (bottom left), and $I_{250} / I_{500}$ (bottom right). The ratios in each image are color coded according to the color bar shown on top of the image. The two ellipses marked in each image correspond to the face-on circles of radii of $250^{\prime \prime}$ and $644^{\prime \prime}$, respectively. A pixel of a ratio image is set to zero if either the numerator or denominator image has a zero intensity in that image pixel as defined in Figure 1.

$\mathrm{H}$ II region) corresponds to an $\mathrm{X}$-axis reading of less than 0.3 . As a result, the warm dust emission associated with the current star formation in M 81 contributes to less than $23 \%$ of the total observed flux at $250 \mu \mathrm{m}$ based on Figure 4. At $500 \mu \mathrm{m}$, this contribution should be less than $\sim 13 \%$. In the remainder of this paper, we regard $I_{500}$ as being only associated with large dust grains heated by evolved stars.

Figure 2 also reveals an apparent anti-correlation between either $I_{8} / I_{\mathrm{H} \alpha}$ or $I_{24} / I_{\mathrm{H} \alpha}$ and $I_{\mathrm{H} \alpha} / I_{500}$, in the sense that local peaks in the last ratio correspond to local dips in either of the former ratios, suggesting that there are factors other than $I_{\mathrm{H} \alpha}$ in powering $I_{8}$ or $I_{24}$. In particular, both the $I_{8} / I_{\mathrm{H} \alpha}$ and $I_{24} / I_{\mathrm{H} \alpha}$ images have peaks in the bulge/inner disk region, between the outer disk, where the major spiral arms and prominent $\mathrm{H}$ II regions reside, and the circumnuclear region, where an enhanced $\mathrm{H} \alpha$ emission is seen. This bulge/inner disk area is devoid of discrete $\mathrm{H} \alpha$ emission of high surface brightness, yet the original high-resolution IRAC $8 \mu \mathrm{m}$ image clearly shows 


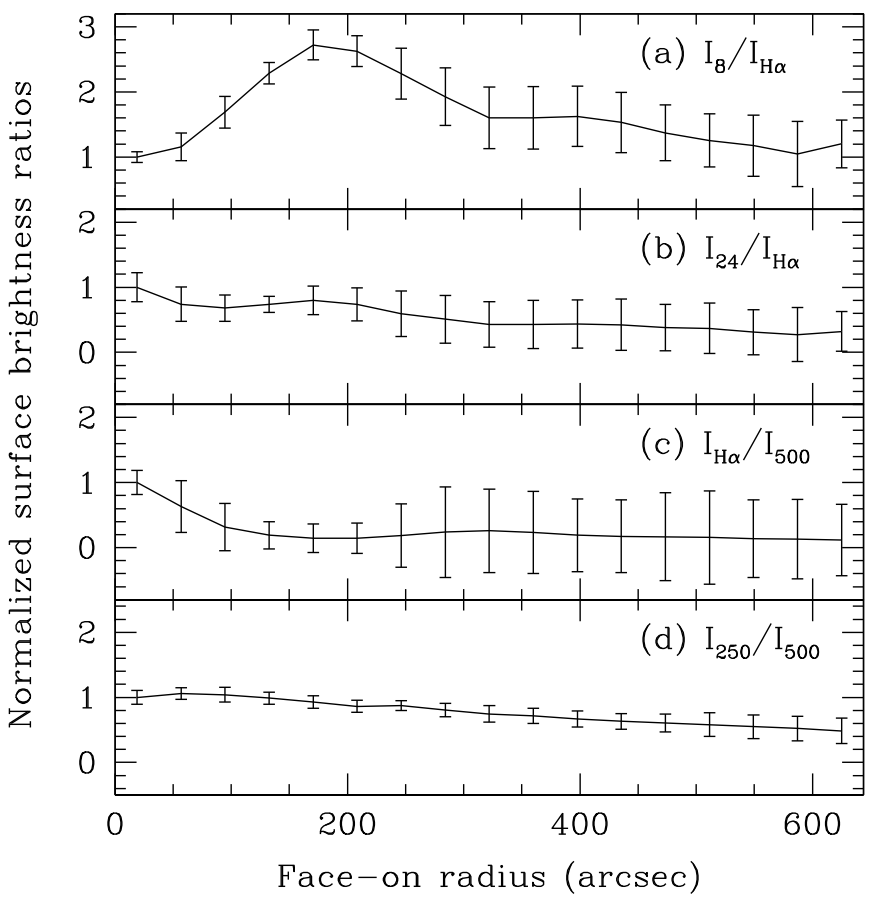

Figure 3. Radial plots of the azimuthally averaged surface brightness ratios: (a) $I_{8} / I_{\mathrm{H} \alpha}$, (b) $I_{24} / I_{\mathrm{H} \alpha}$, (c) $I_{\mathrm{H} \alpha} / I_{500}$, and (d) $I_{250} / I_{500}$. Each radial profile has been normalized by its average in the inner most radial bin. The error bars shown are the sample standard deviation normalized by the actual observed average in the corresponding radial bin. The plots have the same vertical range so that the relative ratio variations can be visually compared among these plots.

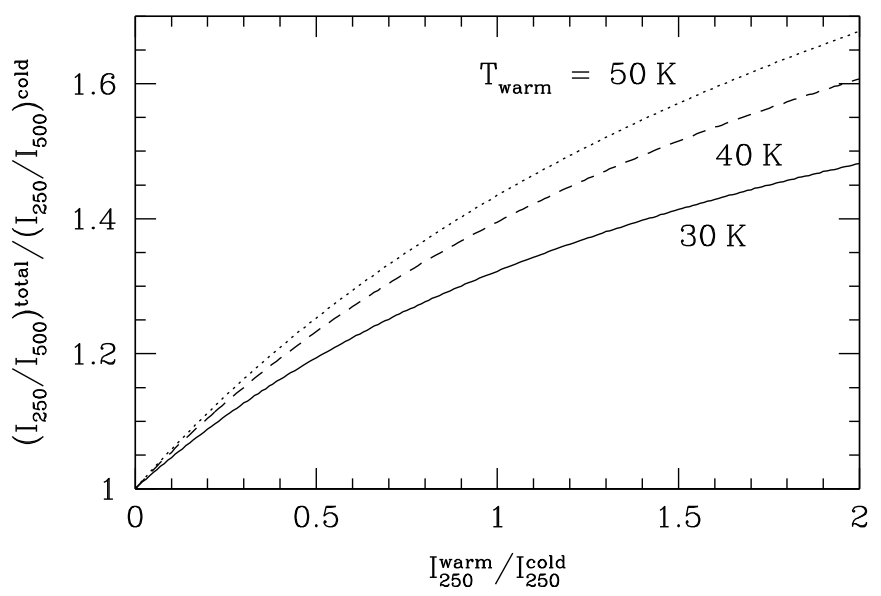

Figure 4. Plot of the enhancement to $I_{250} / I_{500}$ from a warm dust emission of temperature $T_{\text {warm }}$ over a cold dust emission of $T_{\text {cold }}=16 \mathrm{~K}$, as a function of the warm-to-cold surface brightness ratio at $250 \mu \mathrm{m}$. We assumed a common dust emissivity that scales with $v^{2}$. As labeled, the plotted curves used $T_{\text {warm }}=30$, 40 , or $50 \mathrm{~K}$.

PAH emission in spiral patterns (see Willner et al. 2004). Taken at face value, the ratio images in Figure 2 suggest that evolved stars play a significant role in heating both PAHs and VSGs in the case of M 81.

\subsection{Correlation Analysis}

We can analyze Figure 2 in a more quantitative way via Equations (2)-(4). To do so, we first resampled all the images to a pixel size of $36^{\prime \prime}$, roughly equal to the spatial resolution of the SPIRE $500 \mu \mathrm{m}$ band. This is to ensure that individual image pixels are statistically independent from each other. With all the images having the same flux units of Jy pixel ${ }^{-1}$, Figure 5

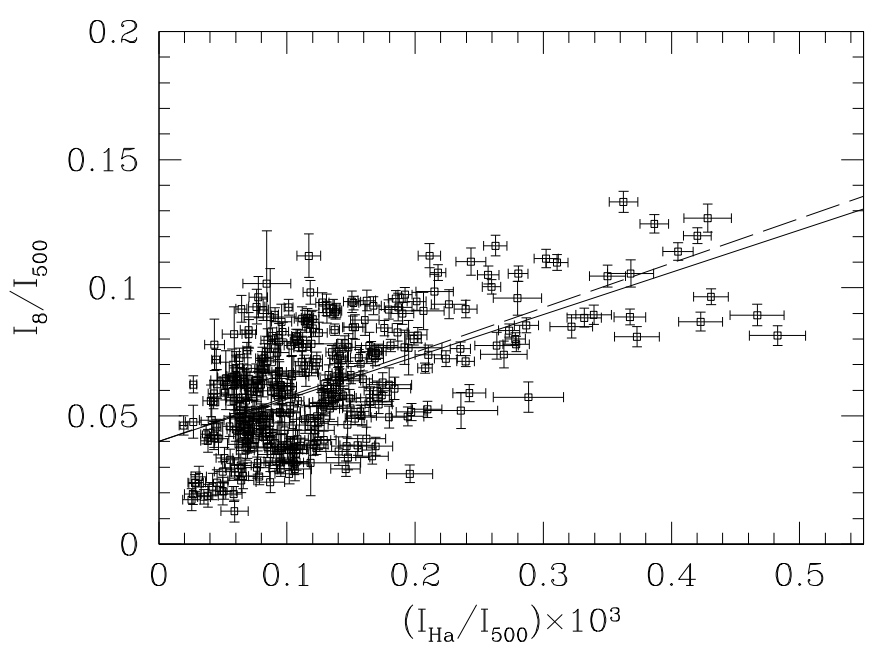

Figure 5. Plot of $I_{8} / I_{500}$ vs. $I_{\mathrm{H} \alpha} / I_{500}$ for all the $36^{\prime \prime}$ pixels in the outer disk between the face-on radii of $250^{\prime \prime}$ and $644^{\prime \prime}$, for which all the involved surface brightness values are greater than three times their respective image background noise. The error bars shown are at $1 \sigma$. The solid and dashed lines are least-squares fits from a simple regression minimizing the residuals along the vertical axis and a full regression that minimizes the residuals along both axes, respectively.

is a simple pixel plot of $I_{8} / I_{500}$ versus $I_{\mathrm{H} \alpha} / I_{500}$ using all those $36^{\prime \prime}$ image pixels between $R_{\text {face-on }}$ of $250^{\prime \prime}$ and $644^{\prime \prime}$, for which the three surface brightness values involved are all above three times their corresponding background noise. This outer disk hosts prominent $\mathrm{H}$ II regions, along the major spiral arms, which should provide a significant or even dominant heating source for the mid-IR dust emissions. Another practical consideration of using only the outer disk in Figure 5 is to maintain more or less the same (and finite) range in the values of $K(T)$ between the diffuse regions and the $\mathrm{H}$ II-dominated regions, in order to obtain an unbiased slope from fitting Equations (2) and (4) to the data.

Indeed, the outer-disk data points in Figure 5 span a range of a factor of $\gtrsim 10$ in terms of $I_{\mathrm{H} \alpha} / I_{500}$. In contrast, $I_{250} / I_{500}$ remains smooth and varies only by a factor of $\sim 2$ (see Figure 2 ). We therefore applied least-squares fits of Equation (2) to the data points in Figure 5, resulting in the two lines shown. The solid line is from a simple regression by minimizing the vertical residuals, while the dotted line is from a full regression by minimizing the residuals along both axes. Within the uncertainties, both the fits agree with each other and can be represented by the following result:

$$
I_{8}=(0.17 \pm 0.01) \times 10^{3} I_{\mathrm{H} \alpha}+(0.040 \pm 0.002) I_{500} .
$$

We also carried out the same analysis on the $24 \mu \mathrm{m}$ emission in Figure 6 via Equation (4). Figure 6 is similar to Figure 5, but uses $I_{24} / I_{500}$ instead. The least-squares fits shown in the figure can be represented by

$$
I_{24}=(0.27 \pm 0.01) \times 10^{3} I_{\mathrm{H} \alpha}+(0.021 \pm 0.002) I_{500} .
$$

It is interesting to see, via Equations (6) and (7), how the warm and cold dust components compare with each other at different locations in the outer disk of the galaxy. Table 2 compares the fractional flux contributions at 8 or $24 \mu \mathrm{m}$ from the warm dust component between two locations that differ by a factor of 10 in terms of $I_{\mathrm{H} \alpha} / I_{500}$. As is evident in Figures 5 and 6 , these two locations represent a diffuse region with the lowest and a bright $\mathrm{HI}$ region with nearly the highest values of $I_{\mathrm{H} \alpha} / I_{500}$ 


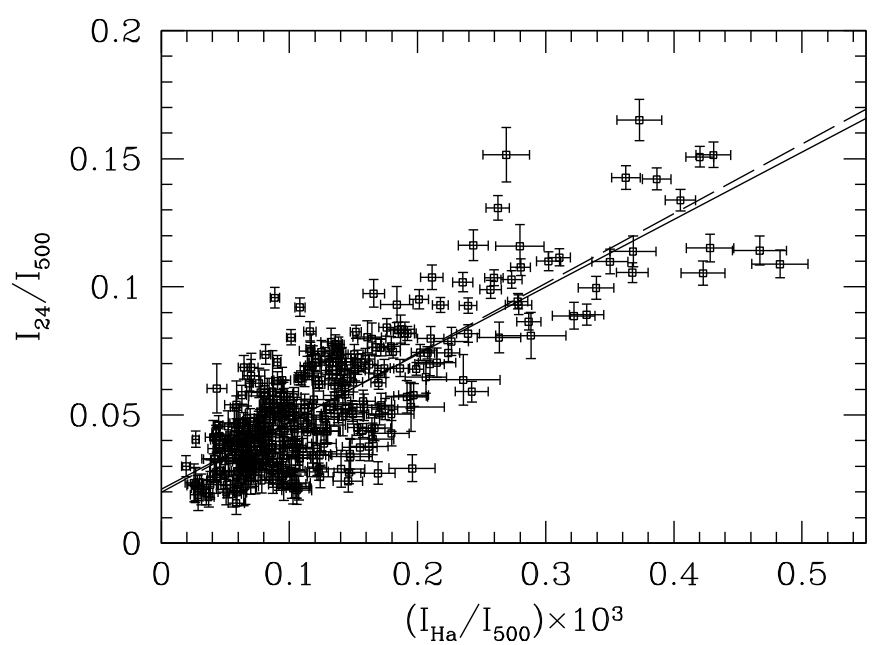

Figure 6. Same as in Figure 5, but of $I_{24} / I_{500}$ vs. $I_{\mathrm{H} \alpha} / I_{500}$.

Table 2

Fractional Flux Contributions from the Warm Dust Component

\begin{tabular}{lccc}
\hline \hline Region & $I_{\mathrm{H} \alpha} / I_{500}$ & $I_{8}($ warm $) / I_{8}($ tot $)$ & $I_{24}($ warm $) / I_{24}($ tot $)$ \\
\hline Diffuse & $0.04 \times 10^{-3}$ & $0.15( \pm 0.01)^{\mathrm{a}}$ & $0.34( \pm 0.02)^{\mathrm{a}}$ \\
H II & $0.40 \times 10^{-3}$ & $0.63( \pm 0.02)^{\mathrm{a}}$ & $0.84( \pm 0.01)^{\mathrm{a}}$ \\
\hline
\end{tabular}

Note. a The errors were estimated using the parameter uncertainties in Equations (6) and (7).

observed, respectively. For the $8 \mu \mathrm{m}$ emission, the fractional flux contribution from the warm component varies from $15 \%$ in such a diffuse region to $63 \%$ in such a bright $\mathrm{H}$ iI region; for the $24 \mu \mathrm{m}$ emission, this variation is $34 \%-84 \%$. This shows clearly that the current star formation dominates both PAH and $24 \mu \mathrm{m}$ dust emissions in or around those bright $\mathrm{H}_{\mathrm{II}}$ regions in $\mathrm{M} 81$. In contrast, in the diffuse region considered in Table 2, this star formation component only accounts for $\sim 15 \%$ and $34 \%$ of the total flux at 8 and $24 \mu \mathrm{m}$, respectively.

\subsection{Variations in $K(T)$}

The scatter in Figures 5 and 6 is much larger than what the statistical errors (shown in the figure) can account for. Figure 7 demonstrates that much of this scatter is due to the variation of the factor $K(T)$, defined in Equation (3), by showing the image pixels in plots of the normalized $I_{8} / I_{500}$ or $I_{24} / I_{500}$ as a function of $I_{250} / I_{500}$, where the normalization was done by dividing the observed ratio by the mean value given in Equations (6) and (7). For example, $\left(I_{8} / I_{500}\right)_{\text {normalized }}=$ $\left(I_{8} / I_{500}\right) /\left(0.17 \times 10^{3} I_{\mathrm{H} \alpha} / I_{500}+0.040\right)$. Therefore, an image pixel lying above (or below) the mean fit in Figure 5 would have the normalized ratio $\left(I_{8} / I_{500}\right)_{\text {normalized }}>1$ (or $\left.<1\right)$. In Figure 7 , we showed the image pixels from both the outer disk (i.e., the squares in the figure) and inner disk (crosses) delineated at $R_{\text {face-on }}=250^{\prime \prime}$. Again, only those image pixels with the appropriate surface brightnesses three times greater than their respective uncertainties are plotted here. For the outer disk data points, as $I_{250} / I_{500}$ [or $\left.K(T)\right)$ ] increases, the normalized $I_{8} / I_{500}$ or $I_{24} / I_{500}$ ratio increases from being $<1$ to $>1$. This trend contributes to much of the scatter seen in Figures 5 and 6 . If we had included the inner disk image pixels in Figures 5 and 6, these data points would be farther above our mean fit to the outer disk data points as the inner data points have even larger values of $K(T)$. In principle, one can divide a galaxy disk into successive

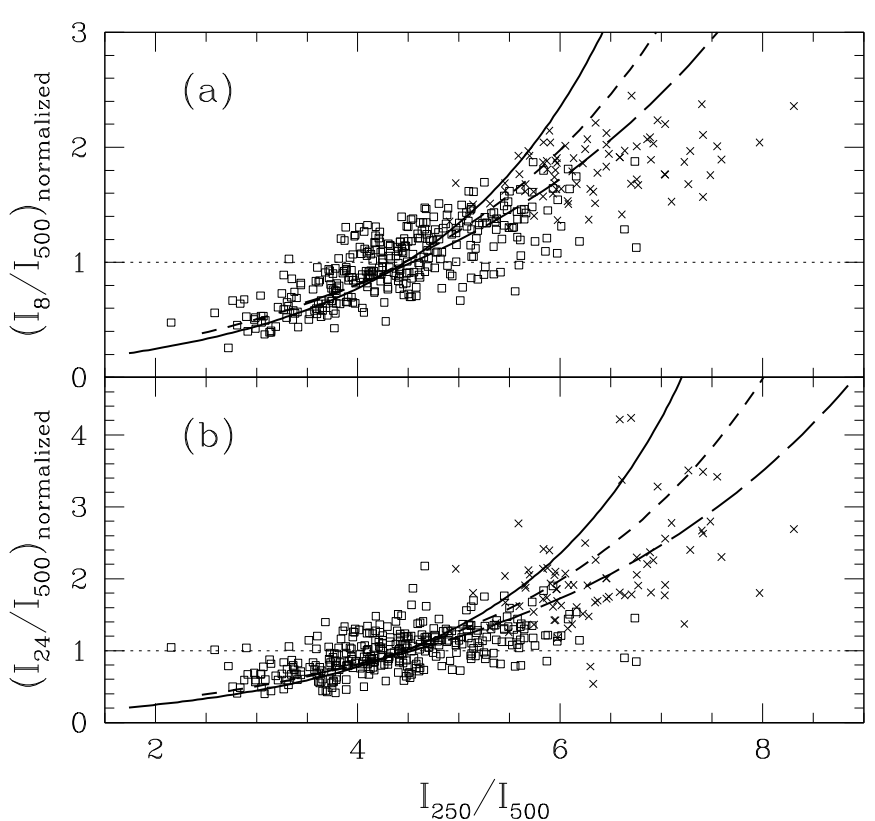

Figure 7. Plots of the image pixels of (a) $I_{8} / I_{500}$ and (b) $I_{24} / I_{500}$, each normalized by their corresponding mean value given by Equations (6) and (7), as a function of $I_{250} / I_{500}$. Those from the outer and inner parts of the galaxy disk, divided at the face-on circle of radius $R_{\text {face-on }}=250^{\prime \prime}$, are shown by squares and crosses (red in the online journal), respectively. Only those pixels with all the involved surface brightnesses three times greater than their respective uncertainties are plotted. The solid, short-dashed, and long-dashed curves are predictions from a Planck function modified by an emissivity that scales with frequency $v$ as $v^{\beta}$ for $\beta=2,2.5$, and 3 , respectively. The left end of each model curve corresponds to the lower limit of $10 \mathrm{~K}$ in dust temperature. All the curves were normalized to unity at $I_{250} / I_{500}=4.5$. The horizontal dotted line marks the location of the the mean fit of Equations (6) and (7).

radial annuli and fit Equations (2) and (4) to individual annuli independently as long as $I_{\mathrm{H} \alpha} / I_{500}$ in each annulus covers enough dynamic range to make such a fit feasible. The fit from each radial annulus, of a different average value of $K(T)$, should give more or less the same slope if the scaling factor " $a$ " in Equation (2) (or "c" in Equation (4)) does not depend on the galactocentric radius. In the case of M 81, it is not feasible to make a separate two-component analysis in the inner disk which contains far fewer image pixels that are also distributed over a much larger range in $K(T)$ (as evidenced by a larger vertical spread for the inner disk data points in Figure 7).

The three curves in each plot of Figure 7 correspond to the predicted $K(T)$ values from a Planck function modified with a dust emissivity $\propto v^{\beta}$ with $\beta=2,2.5$, and 3 , respectively. These values were simply chosen for illustration although the $\beta=2$ case was favored in the SED analysis of Bendo et al. (2010). The left end of each curve corresponds to a dust temperature of $10 \mathrm{~K}$. For the $\beta=2$ model, the dust temperatures are 12.6, 14.7, $17.0,19.5,22.5$, and $26.0 \mathrm{~K}$ at $I_{250} / I_{500}=3,4,5,6,7$, and 8 , respectively. The similar temperature sets for the $\beta=2.5$ and 3 models are $(10.8,12.3,13.7,15.2,16.9,18.6 \mathrm{~K})$ and $(\mathrm{N} / \mathrm{A}$, $10.5,11.6,12.6,13.7,14.7 \mathrm{~K})$, respectively. All the curves are normalized to unity at $I_{250} / I_{500}=4.5$.

While none of these curves can fit the data points over the entire galaxy, they do follow the trend of the data points in the outer disk, consistent with our claim that a large part of the scatter seen in Figures 5 and 6 can be accounted for by the variation in $K(T)$. The inner disk data points (i.e., the crosses in Figure 7) tend to fall below the model curves. This is not necessarily suggestive of a greater value of $\beta$. As 
Table 3

Flux Densities of Cold and Warm Dust Components

\begin{tabular}{|c|c|c|c|}
\hline (1) & $\begin{array}{c}\text { Total } \\
(\mathrm{Jy}) \\
(2)\end{array}$ & $\begin{array}{c}\text { Warm } \\
\text { (Jy) } \\
(3)\end{array}$ & $\begin{array}{c}\text { Cold } \\
(\mathrm{Jy}) \\
(4)\end{array}$ \\
\hline \multicolumn{4}{|c|}{ Outer disk ${ }^{\mathrm{a}}$ : } \\
\hline $8 \mu \mathrm{m}$ & 3.58 & $1.31( \pm 0.08)$ & $2.20( \pm 0.11)$ \\
\hline $24 \mu \mathrm{m}$ & 3.22 & $2.08( \pm 0.08)$ & $1.15( \pm 0.11)$ \\
\hline $\mathrm{H} \alpha$ & $0.0077^{\mathrm{b}}$ & $0.0077^{\mathrm{b}}$ & 0.0 \\
\hline $500 \mu \mathrm{m}$ & 54.96 & 0.0 & 54.96 \\
\hline \multicolumn{4}{|c|}{ Total galaxy ${ }^{\mathrm{c}}$ : } \\
\hline $8 \mu \mathrm{m}$ & 5.03 & 1.65 & 3.38 \\
\hline $24 \mu \mathrm{m}$ & 5.01 & 2.62 & 2.39 \\
\hline $\mathrm{H} \alpha$ & $0.0097^{\mathrm{b}}$ & $0.0097^{\mathrm{b}}$ & 0.0 \\
\hline $500 \mu \mathrm{m}$ & 67.07 & 0.0 & 67.07 \\
\hline
\end{tabular}

\section{Notes.}

a Defined to be the disk between the face-on radii of $250^{\prime \prime}$ and $644^{\prime \prime}$.

b The surface brightness of the $\mathrm{H} \alpha$ image is defined to be the line flux divided by the rest-frame line frequency of $4.57 \times 10^{14} \mathrm{~Hz}$.

c Defined to be the galaxy within the face-on radius of $644^{\prime \prime}$.

further discussed in Section 5, this could also be a result of a greater contamination of the [N II] emission in our $\mathrm{H} \alpha$ flux within the inner disk, where a significantly higher metallicity was observed.

\subsection{Total Fluxes}

We derived a total galaxy flux in each image by summing over all image pixels within $R_{\text {face-on }}=644^{\prime \prime}$, including those with a surface brightness less than three times the sky noise. Similarly, an integrated flux for the outer disk of $250^{\prime \prime}<R_{\text {face-on }} \leqslant 644^{\prime \prime}$ was also derived. These fluxes are shown in Column 2 of Table 3. For the outer disk, Equations (6) and (7) were used to derive the 8 and $24 \mu \mathrm{m}$ fluxes for the warm and cold components, given in Columns 3 and 4, respectively. The associated errors were based on the parameter uncertainties in Equations (6) and (7). Since Equations (6) and (7) were fit to this outer disk, it is not surprising that the sum of the warm and cold component fluxes in the table agrees with the measured total flux in Column 2 for either the 8 or $24 \mu \mathrm{m}$ emission. These results show that, in the outer disk of $\mathrm{M} 81,63 \%( \pm 2 \%)$ of the PAH emission is not directly associated with the current star formation. For the $24 \mu \mathrm{m}$ emission, this fraction is $35 \%( \pm 2 \%)$.

To derive the flux of the cold or warm dust component for the total galaxy, we also need to calculate the contribution from the inner disk/bulge region with $R_{\text {face-on }}<250^{\prime \prime}$. To this end, we assumed that the scaling constant for the warm component in Equations (6) and (7) remains valid in the inner disk/bulge region, and estimated the contribution from the current star formation to $I_{8}$ or $I_{24}$ using the observed $\mathrm{H} \alpha$ flux. Because of this additional assumption, the results for the inner disk region may be subject to additional systematics in the $\mathrm{H} \alpha$ flux as discussed in Section 5. We therefore listed in Table 3 the results for the outer disk separately from those for the entire galaxy. In the later case, $\sim 67 \%$ of the total PAH emission in M 81 is not directly associated with the current star formation. For the $24 \mu \mathrm{m}$ emission, this fraction is $\sim 48 \%$. Clearly, the inclusion of the inner disk and the bulge, where the surface density of evolved stars is high, reduces the overall contribution from the current star formation.

\section{ASSESSMENT OF SYSTEMATICS}

As we mentioned in Section 1, our analysis improved over many of the previous studies on the same subject in two areas: (1) we used a spectrally integrated flux of the cold dust emission instead of a monochromatic flux density; (2) we made no assumption on whether the $3.6 \mu \mathrm{m}$ flux density is a good proxy for the dust heating from evolved stars. ${ }^{17}$ Furthermore, our analysis is empirical, without prior assumption for the dust emissivity law or dust temperatures. This differs from most conventional SED analyses in the literature. On the other hand, our analysis anchored on two basic requirements and may be subject to some systematics. We discuss these two issues separately below.

As introduced in Section 1, our first basic requirement is that $I_{500}$ is dominated by the emission from large dust grains heated by evolved stars. This was shown to be the case in Section 4.1 in this paper and in Bendo et al. (2012). Furthermore, we also require that the (extinction-corrected) $\mathrm{H} \alpha$ flux scales linearly with the far-UV photon flux that dominates the heating of warm dust. This requirement translates to (1) a well mixed gas and dust over our spatial resolution element $\left(\sim 36^{\prime \prime}\right.$ or $\left.0.64 \mathrm{kpc}\right)$ and (2) a fixed initial mass function (IMF) of the current star formation. While this remains as an assumption in principle in this paper, it is reasonable based on the observations that the $\mathrm{PAH}$ and $\mathrm{H} \alpha$ fluxes scale almost linearly with each other for individual $\mathrm{H}$ II regions in nearby normal galaxies (e.g., Calzetti et al. 2007). Nevertheless, even if the IMF varies from one star forming region to another, our results should remain statistically valid for the outer disk of M 81. This also holds true if the PAHto-large dust grain abundance ratio varies across the galaxy disk.

In contrast, our analysis results may be subject to a number of potential systematics in the $\mathrm{H} \alpha$ flux as we already alluded to in Section 3.3. In the outer disk, the internal optical extinction follows gas/dust distribution and generally peaks around bright $\mathrm{H}$ II regions, where the largest surface brightness of the $\mathrm{H} \alpha$ emission is observed. As a result, if we had applied some internal extinction correction to the $\mathrm{H} \alpha$ fluxes, the fit of Equations (6) and (7) to the data would be somewhat flatter, making its $Y$-axis intercept even greater in Figures 5 and 6. In other words, any correction for the internal extinction on $I_{\mathrm{H} \alpha}$ would likely result in an even greater cold component in $I_{8}$ or $I_{24}$.

Another possible systematic issue is the elevated metallicity in the galaxy bulge. If we had taken into account the fact that the [N II] line might be relatively brighter within the galaxy bulge, the warm component fluxes for the inner disk, thus for the total galaxy in Column 3 of Table 3, would have been somewhat overestimated. As a result, the counterpart fluxes of the cold component in Column 4 would have been underestimated accordingly.

Furthermore, a potential controversy exists regarding whether the circumnuclear $\mathrm{H} \alpha$ emission in M 81 is derived from current star formation (see Devereux et al. 1995). If it is not related to the ongoing star formation, the inner disk contribution to the warm dust components in Column 3 in Table 3 should be further reduced, making the overall cold dust component even more significant.

In summary, all these potential systematics in the $\mathrm{H} \alpha$ image can only imply that our estimates of the cold-component

\footnotetext{
17 The $3.6 \mu \mathrm{m}$ flux density was used here to only estimate the stellar continuum at $8 \mu \mathrm{m}$. At wavelengths longer than $\sim 3.6 \mu \mathrm{m}$, the spectral shape of the stellar continuum of a normal galaxy depends little on star formation history or metallicity (Helou et al. 2004). From 8 to $24 \mu \mathrm{m}$, our simple Rayleigh-Jeans approximation of the stellar continuum should be sufficient.
} 
fractional fluxes at both 8 and $24 \mu \mathrm{m}$ in Table 3, especially in the inner disk/bulge region, might be somewhat underestimated.

\section{DISCUSSION}

Our correlation analysis showed that the $24 \mu \mathrm{m}$ emission from VSGs is indeed a better SFR tracer than the $8 \mu \mathrm{m}$ PAH emission as the former is $60 \%$ more sensitive to ionizing stars and $50 \%$ less sensitive to evolved stars than the latter, based on the slopes in Equations (6) and (7). In fact, Table 3 shows that the $24 \mu \mathrm{m}$ flux in the outer disk of M 81 is indeed modestly dominated by the current star formation. This is in agreement with the latest consensus in the literature. On the other hand, $I_{24}$ still derives nearly half of its emission from the general ISM in the case of M 81 when integrated over the galaxy surface, suggesting that even the $24 \mu \mathrm{m}$ emission could arise significantly from VSGs heated by evolved stars for a galaxy as whole. The relatively large stellar mass of M81 may play a role, as implied by studies of other early-type disk galaxies (e.g., Sauvage \& Thuan 1992; Engelbracht et al. 2010). Tabatabaei \& Berkhuijsen (2010) reached a similar conclusion on the heating source for the 24 $\mu \mathrm{m}$ emission in M 31, another nearby IR-quiescent disk galaxy.

It is interesting to compare our M 81 results with the statistical results from analyzing a sample of IRAS galaxies in Lu \& Helou (2008). Their statistical conclusion is that, for galaxies with quiescent FIR colors similar to M 81, most of the PAH emission should be from the cold component, in agreement with the results in the current paper. Furthermore, Lu \& Helou (2008) showed that the fraction of the PAH luminosity that is directly correlated with current star formation increases on average as FIR color increases. In contrast, Kennicutt et al. (2009) showed that nature may present us with a constant fractional warm PAH or $8 \mu \mathrm{m}$ dust emission over a variety of galaxies with FIR colors from 0.3 to 1 , morphological types of $\mathrm{S} 0$ or later, and total IR luminosities as high as $10^{11.9} L_{\odot}$. With an FIR color of $\sim 0.26$, M 81 is at the cold end of the color range occupied by the galaxies used in Kennicutt et al. (2009). It is therefore of great interest to apply the same two-component analysis from this paper to additional galaxies of warmer FIR colors.

Many starburst dwarf galaxies show a depressed PAH emission (e.g., Engelbracht et al. 2005; Hogg et al. 2005). The reason could be either intrinsic, i.e., a low carbon-based PAH abundance relative to silicon-based large dust grains (e.g., Galliano et al. 2008) or extrinsic, e.g., via PAH destruction by hard UV or supernova shocks associated with massive stars (e.g., Madden et al. 2006; O'Halloran et al. 2006; Engelbracht et al. 2008). While the latter explanation is more widely accepted today, the former has not been convincingly ruled out. It is difficult to differentiate these two scenarios observationally because the IR emission in star forming dwarf galaxies is always dominated by their high surface brightness $\mathrm{H}_{\mathrm{II}}$ regions. The methods laid out in this paper could in principle be used to differentiate these two scenarios: The mean slope $A$ in Equation (1) could vary from galaxy to galaxy because (1) dust mass spectrum may vary (e.g., via metallicity change) or/and (2) PAH may be destroyed to various degrees (as the hardness of UV radiation field may vary). In contrast, the mean value of $B$ in Equation (1) should depend only on (1). It is therefore possible to statistically compare samples of dwarfs and normal spirals to see if there is a meaningful difference in the fitted parameter $B$ between the two galaxy classes. Such an analysis is now feasible with Herschel SPIRE data of many low-metallicity dwarf galaxies (Madden et al. 2013).

\section{CONCLUSION}

Using the newly available Herschel images at 250 and $500 \mu \mathrm{m}$ and the existing Spitzer images at 8 and $24 \mu \mathrm{m}$ and ground-based $\mathrm{H} \alpha$ image, we demonstrated a new, two-component correlation analysis method that is applicable to galaxy images of any angular resolution and takes into consideration the frequencyintegrated spectrum of the cold dust emission.

Using this method, we showed that, in the case of M81, the surface brightnesses of both the $8 \mu \mathrm{m}$ emission from PAHs and the $24 \mu \mathrm{m}$ emission from VSGs can be quantitatively decomposed into two components, free from any model-dependent assumptions: (1) a component that scales with the surface brightness of the $\mathrm{H} \alpha$ line emission, which is a well known tracer of current star formation rate; and (2) a component that scales with the surface brightness of the cold, diffuse dust emission at $500 \mu \mathrm{m}$, which is known to be heated primarily by evolved stars. Roughly, the cold components constitute about $67 \%$ and $48 \%$ of the observed 8 and $24 \mu \mathrm{m}$ emissions, respectively.

We argued that these estimates on the fractional cold dust component likely represent a lower limit if any of the following uncorrected systematics in our $\mathrm{H} \alpha$ image is significant: patchiness in the internal extinction, non-stellar origin of the nuclear $\mathrm{H} \alpha$ emission, and an elevated [N $\mathrm{NI}_{\mathrm{II}}$ contamination to the $\mathrm{H} \alpha$ line flux within the galaxy bulge.

We thank the anonymous referee for a number of useful comments that improved the presentation and clarity of this paper. This research has made use of the NASA/IPAC Extragalactic Database (NED) which is operated by the Jet Propulsion Laboratory, California Institute of Technology, under contract with the National Aeronautics and Space Administration. SPIRE has been developed by a consortium of institutes led by Cardiff University (UK) and including Univ. Lethbridge (Canada); NAOC (China); CEA, OAMP (France); IFSI, Univ. Padua (Italy); IAC (Spain); Stockholm Observatory (Sweden); Imperial College London, RAL, UCL-MSSL, UKATC, Univ. Sussex (UK); and Caltech/JPL, IPAC, Univ. Colorado (USA). This development has been supported by national funding agencies: CSA (Canada); NAOC (China); CEA, CNES, CNRS (France); ASI (Italy); MCINN (Spain); Stockholm Observatory (Sweden); STFC (UK); and NASA (USA).

\section{REFERENCES}

Allamandola, L. J., Tielens, A. G. G. M., \& Barker, J. R. 1989, ApJS, 71, 733 Bendo, G. J., Boselli, A., Dariush, A., et al. 2012, MNRAS, 419, 1833 Bendo, G. J., Draine, B. T., Engelbracht, C. W., et al. 2008, MNRAS, 389, 629 Bendo, G. J., Joseph, R. D., Wells, M., et al. 2003, AJ, 125, 2361 Bendo, G. J., Wilson, C. D., Pohlen, M., et al. 2010, A\&A, 518, L65 Boquien, M., Calzetti, D., Combes, F., et al. 2011, AJ, 142, 111 Boselli, A., \& Gavazzi, G. 2002, A\&A, 386, 124 Boselli, A., Lequeux, J., \& Gavazzi, G. 2004, A\&A, 428, 409 Boulanger, F., Beichman, C., Désert, F. X., et al. 1988, ApJ, 332, 328 Calapa, M. D., Calzetti, D., Draine, B. T., et al. 2014, ApJ, 784, 130 Calzetti, D., Kennicutt, R. C., Engelbracht, C. W., et al. 2007, ApJ, 666, 870 Castelaz, M. W., Sellgren, K., \& Werner, M. W. 1987, ApJ, 313, 853 Chan, K.-W., Roellig, T. L., Onaka, T., et al. 2001, ApJ, 546, 273 Crocker, A. F., Calzetti, D., Thilker, D. A., et al. 2013, ApJ, 762, 79 Davies, J. I., Wilson, C. D., Auld, R., et al. 2010, MNRAS, 409, L102 de Blok, W. J. G., Walter, F., Brinks, E., et al. 2008, AJ, 136, 2648 de Vaucouleurs, G., de Vaucouleurs, A., Corwin, H. G., Jr., et al. 1991, Third Reference Catalog of Bright Galaxies (New York: Springer) Désert, F.-X., Boulanger, F., \& Puget, J. L. 1990, A\&A, 237, 215 Devereux, N. A., Jacoby, G., \& Giardullo, R. 1995, AJ, 110, 1115 Draine, B. T., \& Li, A. 2007, ApJ, 657, 810

Dwek, E., Arendt, R. G., Fixsen, D. J., et al. 1997, ApJ, 475, 565

Engelbracht, C. W., Gordon, K. D., Rieke, G. H., et al. 2005, ApJL, 628, L29 
Engelbracht, C. W., Hunt, L. K., Skibba, R. A., et al. 2010, A\&A, 518, L56

Engelbracht, C. W., Rieke, G. H., Gordon, K. D., et al. 2008, ApJ, 678, 804

Ferguson, A. M. N., Wyse, R. F. G., Gallagher, J. S., III., \& Hunter, D. A. 1996, AJ, 111, 2265

Förster Schreiber, N. M., Roussel, H., Sauvage, M., \& Charmandaris, V. 2004, A\&A, 419, 501

Galliano, F., Dwek, E., \& Chanial, P. 2008, ApJ, 672, 214

Garnett, D. R., \& Shields, G. A. 1987, ApJ, 317, 82

Gordon, K. D., Engelbracht, C. W., Rieke, G. H., et al. 2008, ApJ, 682, 336

Greenawalt, B., Walterbos, R. A. M., Thilker, D., \& Hoopes, C. G. 1998, ApJ, 506,135

Griffin, M. J., Abergel, A., Abreu, A., et al. 2010, A\&A, 518, L3

Haas, M., Klaas, U., \& Bianchi, S. 2002, A\&A, 385, L23

Haffner, L. M., Dettmar, R.-J., Beckman, J. E., et al. 2009, RvMPh, 81, 969

Heckman, T. M. 1980, A\&A, 87, 152

Helou, G., Roussel, H., Appleton, P., et al. 2004, ApJS, 154, 253

Hogg, D. W., Tremonti, C. A., \& Blanton, M. R. 2005, ApJ, 624, 162

Hoopes, C. G., Walterbos, R. A. M., \& Bothun, G. D. 2001, ApJ, 559, 878

Irwin, J. A., \& Madden, S. C. 2006, A\&A, 445, 123

Jones, A. G., Bendo, G. J., Fritz, J., et al. 2014, MNRAS, submitted

Karachentsev, I. D., Dolphin, A. E., Geisler, D., et al. 2002, A\&A, 383, 125

Kaufman, M., Elmegreen, D. M., \& Bash, F. N. 1989, ApJ, 345, 697

Kennicutt, R. C. 1998, ApJ, 498, 541

Kennicutt, R. C., Armus, L., Bendo, G., et al. 2003, PASP, 115, 928

Kennicutt, R. C., Hao, C.-N., Calzetti, D., et al. 2009, ApJ, 703, 1672

Lemke, D., Mattila, K., Lehtinen, K., et al. 1998, A\&A, 331, 742

Lu, N. 2004, ApJS, 154, 286

Lu, N., \& Helou, G. 2008, in ASP Conf. Ser. 381, Infrared Diagnostics of Galaxy Evolution, ed. R.-R. Chary, H. I. Teplitz, \& K. Sheth (San Francisco, CA: ASP), 46
Lu, N., Helou, G., Werner, M., et al. 2003, ApJ, 588, 199

Madden, S. C., Galliano, F., Jones, A. P., \& Sauvage, M. 2006, A\&A, 446, 877

Madden, S. C., Rémy-Ruyer, A., Galametz, M., et al. 2013, PASP, 125,600

Mathis, J. S. 1986, ApJ, 301, 423

Mattila, K., Juvela, M., \& Lehtinen, K. 2007, ApJL, 654, L131

Mentuch, E., Abraham, R. G, \& Zibetti, S. 2010, ApJ, 725, 1971

O'Halloran, B., Satyapal, S., \& Dudik, R. P. 2006, ApJ, 641, 795

Perelmuter, J.-M., \& Racine, R. 1995, AJ, 109, 1055

Pilbratt, G., Riedinger, J. R., Passvogel, T., et al. 2010, A\&A, 518, L1

Puget, J. L., \& Léger, A. 1989, ARA\&A, 27, 161

Reynolds, R. J. 1991, in IAU Symp. 144, The Interstellar DiskHalo Connection in Galaxies, ed. H. Bloemen (Dordrecht: Kluwer), 67

Rice, W., Lonsdale, C. J., Soifer, B. T., et al. 1988, ApJS, 68, 91

Roussel, H., Sauvage, M., Vigroux, L., \& Bosma, A. 2001, A\&A, 372, 427

Sánchez-Gallego, J. R., Knapen, J. H., Wilson, C. D., et al. 2012, MNRAS, 422, 3208

Schmidt, M. 1959, ApJ, 129, 243

Sauvage, M., \& Thuan, T. X. 1992, ApJ, 396, 69

Schroder, L. L., Brodie, J. P., Kissler-Patig, M., Huchra, J. P., \& Phillips, A. C. 2002, AJ, 123, 2473

Tabatabaei, F. S., \& Berkhuijsen, E. M. 2010, A\&A, 517, 77

Uchida, K. I., Sellgren, K., \& Werner, M. 1998, ApJL, 493, L109

Walterbos, R. A. M., \& Braun, R. 1994, ApJ, 431, 156

Willner, S. P., Ashby, M. L. N., Barmby, P., et al. 2004, ApJS, 154, 222

Witt, A. N., Gold, B., Barnes, F. S., III., et al. 2010, ApJ, 724, 1551

Wood, K., \& Reynolds, R. J. 1999, ApJ, 525, 799

Wu, H., Cao, C., Hao, C.-N., et al. 2005, ApJL, 632, L79

Xilouris, E. M., Tabatabaei, F. S., Boquien, M., et al. 2012, A\&A, 543, 74

Zhu, Y.-N., Wu, H., Cao, C., \& Li, H.-N. 2008, ApJ, 686, 155 